\title{
Predictors of postoperative urinary retention after posterior spinal fusion for adolescent idiopathic scoliosis
}

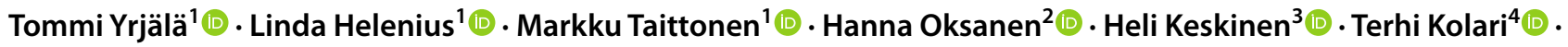 \\ Ilkka Helenius ${ }^{5}$ iD
}

Received: 12 April 2021 / Revised: 26 August 2021 / Accepted: 28 September 2021 / Published online: 5 October 2021

(c) The Author(s) 2021

\begin{abstract}
Purpose To determine predictors for postoperative urinary retention in adolescents undergoing posterior spinal fusion for idiopathic scoliosis. Postoperative urinary retention affects almost every third adolescent after spinal fusion for idiopathic scoliosis. There are limited data regarding the risk factors of postoperative urinary retention in this patient group.

Methods A retrospective study with prospectively collected urinary retention data from paediatric spine register with 159 consecutive patients (114 females, mean age 15.6 years, range 10-21 years) undergoing pedicle screw instrumentation for adolescent idiopathic scoliosis at a university hospital between May 2010 and April 2020. Postoperative urinary retention was defined as an inability to void after catheter removal and documented residual over $300 \mathrm{~mL}$ as confirmed using an ultrasound or by catheterization.

Results Postoperative urinary retention was diagnosed in 33\% (53 of 159) of the patients during hospital stay. Opioid amount on the day of catheter removal (OR 6.74 [95\% CI: 2.47, 18.36], $p<0.001$ ), male gender (OR 2.26 [95\% CI: 1.01, 5.05], $p=0.048$ ), and increasing weight (OR 1.04 [95\% CI: 1.01, 1.07], $p=0.014$ ) were associated with postoperative urinary retention. Mean opioid consumption on the day of catheter removal was $0.81 \mathrm{mg} / \mathrm{kg}(95 \% \mathrm{CI}: 0.66,0.96)$ in the retention group vs $0.57 \mathrm{mg} / \mathrm{kg}(95 \% \mathrm{CI}: 0.51,0.64)$ in the non-retention group, $p<0.001$.

Conclusions Higher total opioid consumption, opioid amount on the day of catheter removal, higher weight, and male gender increases the risk of postoperative urinary retention in adolescents with idiopathic scoliosis undergoing posterior spinal fusion.
\end{abstract}

Keywords Postoperative urinary retention $\cdot$ Adolescent idiopathic scoliosis $\cdot$ Posterior spinal surgery $\cdot$ Opioid consumption

Tommi Yrjälä

tommi.yrjala@tyks.fi

1 Department of Anaesthesia and Intensive Care, University of Turku and Turku University Hospital, Kiinamyllynkatu 4-8, 20521 Turku, Finland

2 Department of Paediatric Orthopaedic Surgery, University of Turku and Turku University Hospital, Turku, Finland

3 Department of Orthopaedics and Traumatology, University of Turku and Turku University Hospital, Turku, Finland

4 Department of Biostatistics, University of Turku, Turku, Finland

5 Department of Orthopaedics and Traumatology, University of Helsinki and Helsinki University Hospital, Helsinki, Finland

\section{Introduction}

Postoperative urinary retention (POUR) is defined as an inability to void in the presence of a full bladder after surgery or a major residual volume after voiding $[1,2]$. The definition of POUR in children and adolescents varies between studies [1]. POUR can be defined as an inability to void $8 \mathrm{~h}$ after surgery or by clinical symptoms and catheterization or by ultrasound. The reported incidence of POUR in published data is $5-70 \%$ due to multifactorial aetiology and the lack of a uniform definition criteria [1]. The urinary bladder capacity of adults is normally about $300-600 \mathrm{~mL}$ [1, 3-7]. In children the expected urinary bladder capacity is defined by the formula $(30 \times$ [age in years +1$] \mathrm{mL})$, but this is valid only for children aged 4 to 12 years [8]. POUR can lead to increased risk of urinary tract infection, prolonged hospital 
stay, and over distention of the detrusor muscle when left untreated [1-3, 9-11].

Urinary retention is common in AIS patients after posterior spinal fusion (PSF) and affects almost every third of these patients [4]. The published data on predictors of POUR in adolescents are very limited [4, 9]. Additionally, the correction of spinal deformity as a risk factor for POUR remains unclear.

\section{Materials and methods}

The study was approved by the Ethics Committee of Turku University Hospital District.

\section{Study design}

The aim of our study was to evaluate the predictors of POUR in adolescents undergoing pedicle screw instrumentation for AIS. We hypothesized that higher postoperative opioid consumption would increase the risk of POUR.

This is a retrospective study on the incidence and risk factors for POUR with a prospectively collected spine register data including urinary retention. We analysed the data of all consecutive AIS patients surgically treated at our institution with PSF between May 2010 and April 2020. All patients were prospectively screened and treated if necessary, for postoperative urinary retention or difficulties to empty bladder after urinary catheter removal. One hundred and sixtysix consecutive patients (aged 10-21 years) undergoing PSF for AIS using bilateral pedicle screw instrumentation were screened. The diagnosis was adolescent idiopathic scoliosis, and the indication for instrumented PSF was a major curve $45^{\circ}$ or more in Cobb angle [12]. All the procedures were performed at a University Hospital and were done by the same two experienced orthopaedic spine surgeons. Seven patients were excluded from further analyses: two patients did not have enough data on postoperative opioid consumption; two patients needed early re-operation; one patient had additional anterior surgery; one patient had limited renal function, and one patient had neurological comorbidity, leaving 159 patients for further analyses.

Urinary retention was measured by trained nurses using the ultrasound scanner (PadScan HD3), which has in previous studies shown to be reliable in experienced hands [1, $5,13,14]$. Ultrasound scanner was used routinely in every patient at least twice on two separate occasions to measure the residual volume of the urinary bladder. Residual volume was measured after first voiding following catheter removal, and every $4 \mathrm{~h}$ if patient had voiding difficulties or residual over $100 \mathrm{~mL}$ after voiding. Residual volume measuring was discontinued when patients were able to void normally and residual volume after voiding was twice below $100 \mathrm{~mL}$.
POUR was defined as an inability to void after catheter removal and documented full bladder (over $300 \mathrm{~mL}$ residual volume) with ultrasound. In our patient cohort the formula for POUR in children could not be used, since majority of the patients were over 12 years of age. When an over $300 \mathrm{~mL}$ residual was diagnosed by ultrasound and the patients were unable to void, the patients were monitored for max $30 \mathrm{~min}$ and then the patients tried to void again. If the patients still were unable to void, the urinary retention was treated by emptying the bladder by a straight catheter and the urinary volume was measured.

The anaesthetic management of the AIS patients was standardized. The standardized anaesthesia protocol has been used unchanged since 2009. Total intravenous anaesthesia was used in all patients. Anaesthesia was maintained by propofol-TCI, remifentanil-TCI, and dexmedetomidine-infusions. None of the patient required postoperative ventilation.

The majority of the patients $(124 / 159,78 \%)$ had patientcontrolled analgesia with oxycodone for first $48 \mathrm{~h}$ postoperative pain management. The standard oxycodone PCA included on demand oxycodone-bolus of $0.03 \mathrm{mg} / \mathrm{kg} / \mathrm{dose}$ maximally every $10 \mathrm{~min}$ and an hourly maximum of three doses, without basal infusion. The remaining 25 patients received intravenous and oral oxycodone. The oral oxycodone doses of the patients were calculated to equivalent intravenous doses $(0.6 \times$ po dose $)[15,16]$. Oral paraceta$\mathrm{mol} 15-20 \mathrm{mg} / \mathrm{kg}$ three times daily was administered to all patients. None of the patients had epidural analgesia.

The patients were mobilized according to a standardized protocol. On the first postoperative day, the patients were requested to stand up and take a couple of steps. On the second postoperative day, the patients were encouraged to walk on the ward. Urinary catheter was routinely removed on a morning of postoperative day $2-4$ based on patients' clinical condition, and the residual volume was measured with ultrasound on every patient.

Perioperative variables collected include gender, age, height, weight, body mass index (BMI), Lenke classification, fusion levels, intraoperative screw replacement, surgical time, estimated blood loss, length of hospital stay, catheter removal day, urinary volume if catheterized, opioid amount on first $48 \mathrm{~h}$ after operation, opioid amount on the catheter removal day, total opioid consumption during hospital stay.

\section{Surgical technique}

All patients were operated using posterior approach and had spinal cord monitoring (somatosensory evoked potentials [SEP], motor evoked potentials [MEP] and lumbar nerve root electromyography [EMG]). Bilateral segmental pedicle screw instrumentation (MESA 5.5, Stryker spine, 6.35CD Legacy or Solera 6.0, Medtronics Spinal and Biologics, 
Memphis, TN, USA) was used to correct spinal deformity. Free hand technique was used to insert pedicle screws.

\section{Statistical methods}

Continuous variables, which followed normal distribution, were summarized with mean and standard deviation (SD). Skewed distributions were reported with median and lower (Q1) and upper quartiles (Q3). Age was summarized with mean together with range. Categorical variables were summarized with counts $(n)$ and percentages. Background characteristics were tested using Fisher's exact test or analysis of variance (ANOVA). Correlation between continuous variables were carried out calculating Pearson correlation coefficients.

Differences between groups were analysed using binomial logistic regression. Odds ratios (ORs) with 95\% confidence intervals were reported. First univariate analysis was performed. Then all factors were added to the multivariate models and from these models, non-significant factors were gradually omitted. Also interactions between factors were analysed. All tests were performed as two-sided with a significance level set at 0.05 . The analyses were carried out using SAS System, version 9.4 for Windows (SAS Institute Inc., Cary, NC, USA).

\section{Results}

One hundred and fifty-nine patients (114 females [72\%] and 45 males [28\%]) with an average age of 15.6 years (range 10-21 years) at the time of surgery were evaluated. Mean (SD) surgical time was $176 \mathrm{~min}$ (45 min), and median blood loss during surgery was $430 \mathrm{~mL}$ (Q1 280, Q3 660). Mean (SD) number of fused levels was 11.3 (1.9). Mean postoperative day of catheter removal was 2.7 (range 1-5 days). Fiftythree patients (33\%) fulfilled the criteria for POUR, i.e. they were unable to void with full bladder (a residual more than $300 \mathrm{~mL}$ shown by ultrasound) or after voiding the residual volume was over $300 \mathrm{~mL}$ shown by ultrasound. Patients with POUR had a mean of $555 \mathrm{~mL}$ (range $320-1400 \mathrm{~mL}$ ) urinary volume drained at catheterization. Mean time for the first need of catheterization after urinary catheter removal was $6 \mathrm{~h}$ (range $2-16 \mathrm{~h}$ ). Sixteen patients needed only one straight catheterization, and 37 patients needed more than one. Median need for emptying of the bladder by straight catheter was three times (range 1-16). Forty-nine percent (26/53) of the patients needed catheterization more than $24 \mathrm{~h}$ after catheter removal. Patients with POUR had a longer hospital stay than patients without POUR, mean (SD) 7.8 (1.9) days vs $6.8(1.4)$ days $(p<0.001)$.

\section{Predictors of POUR}

Children with POUR had a significantly higher total opioid consumption during hospital stay (mean $4.59 \mathrm{mg} / \mathrm{kg}$ [95\% CI: 3.78, 5.41]) than children not experiencing POUR $(3.38 \mathrm{mg} / \mathrm{kg}$ [95\% CI: 3.02, 3.74]), ( $p=0.002)$. Additionally, patients with POUR received greater opioid amount on the day of catheter removal (mean $0.81 \mathrm{mg} / \mathrm{kg}$ [95\% CI: $0.66,0.96$ ] vs $0.57 \mathrm{mg} / \mathrm{kg}$ [95\% CI: $0.51,0.64], p<0.001)$. Total opioid consumption and opioid amount on the day of the catheter removal had a strong positive correlation $(r=0.83, p<0.001)$.

There was a statistically significant difference between the distributions of some background characteristics between patients with and without POUR. Forty-seven percent $(21 / 45)$ of males were diagnosed with POUR vs $28 \%$ of females $(32 / 82)(p=0.039)$. The surgical time of the patients with POUR was on average $188 \mathrm{~min}$ (SD 49 min) vs patients without POUR 171 min (SD 42 min) $(p=0.029)$. The median blood loss during surgery was $550 \mathrm{~mL}(\mathrm{Q} 1: 350, \mathrm{Q} 3: 830)$ in retention group vs $410 \mathrm{~mL}$ $(\mathrm{Q} 1: 240, \mathrm{Q} 3: 600)$ in non-retention group $(p=0.002)$ (Table 1). Blood loss during surgery explained statistically significantly surgical time $(p<0.001)$. Furthermore, the surgical time predicted significantly both total opioid consumption $(p<0.001)$ and opioid amount on the day of the catheter removal $(p=0.005)$. Higher weight of the patient was associated with increased risk of urinary retention (POUR median $59.9 \mathrm{~kg}$ [Q1:52.2, Q3:72.0] vs nonPOUR 54.5 kg [Q1:48.9, Q3:62.3], $p=0.024)$. Patients' age, height, BMI, Lenke classification, number of catheterization days or 48-h postoperative opioid consumption did not affect the urinary retention outcomes.

Binomial logistic regression analysis for predictors of POUR was performed (Table 2). Univariate analysis indicated that greater total opioid consumption during hospital stay (OR 1.24 [95\% CI: 1.07, 1.45], $p=0.005$ ), greater opioid amount on the day of the catheter removal (OR 3.90 [95\% CI: 1.64, 9.29], $p=0.002$ ), longer surgical time (OR 1.64 [95\% CI: 1.05, 2.58], $p=0.031$ ), male gender (OR 2.24 [95\% CI: 1.10, 4.58], $p=0.027$ ), and increasing weight (OR 1.03 [95\% CI 1.00, 1.06], $p=0.029)$ predicted POUR.

Multivariable logistic regression analysis indicated that greater opioid amount during catheter removal day (OR 6.74 [95\% CI: 2.47, 18.36], $p<0.001$ ), male gender (OR 2.26 [95\% CI: 1.01, 5.05], $p=0.048$ ), and weight (OR 1.04 [95\% CI: $1.01,1.07], p=0.014)$ were associated significantly with POUR.

In the additional multivariate analysis, opioid amount during catheter removal day was replaced by the total opioid consumption during hospital stay. Total opioid consumption 
Table 1 Demographic characteristics in patients with and without postoperative urinary retention (POUR)
Table 2 Univariate binomial logistic regression analysis for postoperative urinary retention

\begin{tabular}{|c|c|c|c|}
\hline Variables & $\begin{array}{l}\text { Patients with } \\
\text { POUR }(n=53)\end{array}$ & $\begin{array}{l}\text { Patients without } \\
\text { POUR }(n=106)\end{array}$ & $p$ value \\
\hline Sex (male : female) & $21: 32$ & $24: 82$ & 0.039 \\
\hline Age (years) & $15.44(2.03)$ & $15.73(2.26)$ & 0.425 \\
\hline Weight (kg) & $61.66(14.36)$ & $56.58(12.65)$ & 0.024 \\
\hline Height (m) & $1.69(0.10)$ & $1.66(0.09)$ & 0.110 \\
\hline BMI $\left(\mathrm{kg} / \mathrm{m}^{2}\right)$ & $21.62(4.19)$ & $20.48(3.72)$ & 0.084 \\
\hline \multicolumn{4}{|l|}{ Lenke classification $(n)$} \\
\hline 1 & 17 & 37 & \\
\hline 2 & 18 & 36 & \\
\hline 3 & 6 & 4 & \\
\hline 4 & 3 & 10 & \\
\hline 5 & 0 & 5 & \\
\hline 6 & 9 & 14 & \\
\hline Instrumentation to L1 or below (n) & $38(71.7 \%)$ & $63(59.4 \%)$ & 0.163 \\
\hline Number of vertebrae fused & $11.45(1.56)$ & $11.17(1.73)$ & 0.317 \\
\hline Screw replacement (n) & $16(30.2 \%)$ & $26(24.5 \%)$ & 0.452 \\
\hline Surgical time (min) & $188(49)$ & $171(42)$ & 0.029 \\
\hline Intraoperative blood loss (mL) & $646(403)$ & $469(304)$ & 0.002 \\
\hline Total oxycodone dose during hospital stay (mg/kg) & $4.59(2.95)$ & $3.38(1.89)$ & 0.002 \\
\hline Oxycodone dose during catheter removal day $(\mathrm{mg} / \mathrm{kg})$ & $0.81(0.54)$ & $0.57(0.32)$ & $<0.001$ \\
\hline $48 \mathrm{~h}$ postoperative oxycodone $(\mathrm{mg} / \mathrm{kg})$ & $1.81(0.90)$ & $1.61(0.70)$ & 0.122 \\
\hline
\end{tabular}

Data presented as mean and standard deviation or number and percentage

\begin{tabular}{llll}
\hline Variables & Odds ratio & $95 \%$ CI & $p$ value \\
\hline Sex & & & \\
$\quad$ Female & Reference & & \\
Male & 2.242 & $1.098-4.579$ & 0.027 \\
Age (years) & 0.939 & $0.805-1.095$ & 0.422 \\
Weight $(\mathrm{kg})$ & 1.028 & $1.003-1.055$ & 0.029 \\
BMI (m/ $\mathrm{kg}^{2}$ ) & 1.075 & $0.989-1.169$ & 0.088 \\
Instrumentation to & & & \\
T12 or above & Reference & & \\
$\quad$ L1 or below & 1.729 & $0.848-3.525$ & 0.132 \\
Number of vertebrae fused & 1.108 & $0.907-1.354$ & 0.315 \\
Screw replacement & 1.331 & $0.638-2.774$ & 0.446 \\
Surgical time (min) & 1.641 & $1.045-2.577$ & 0.031 \\
Intraoperative blood loss $(\mathrm{mL})$ & 1.001 & $1.000-1.002$ & 0.004 \\
Total oxycodone dose during hospital stay $(\mathrm{mg} / \mathrm{kg})$ & 1.244 & $1.070-1.446$ & 0.005 \\
Oxycodone dose on day of catheter removal $(\mathrm{mg} / \mathrm{kg})$ & 3.902 & $1.640-9.285$ & 0.002 \\
48 h postoperative oxycodone $(\mathrm{mg} / \mathrm{kg})$ & 1.387 & $0.911-2.112$ & 0.127 \\
\hline
\end{tabular}

Data presented as odds ratio and $95 \% \mathrm{CI}$ also increased the risk of POUR (OR 1.36 [95\% CI: 1.14, $1.61], p<0.001$ ), as well as male gender (OR 2.22 [95\% CI: $1.00,4.92], p=0.048$ ) and weight (OR 1.04 [95\% CI: 1.01, $1.07], p=0.019)$.

\section{Discussion}

In the current study, 33\% of the patients undergoing posterior spinal fusion for AIS developed postoperative urinary retention after catheter removal. Opioid consumption on 
day of catheter removal and also higher cumulative opioid consumption during hospital stay significantly predicted development of POUR. Other predicting factors included male gender, higher weight, and longer operation time.

There is only one study published with AIS patients undergoing PSF and the relation between opioid consumption and POUR [9]. They claimed that higher opioid consumption on catheter removal day predicted urinary retention. Interestingly, they also found that cumulative opioid dosing on days leading up to catheter removal seemed to be protective against POUR. In their study however, morphine equivalent dose on the day of catheter removal did not differ between urinary retention outcomes in univariate analysis. In binomial logistic regression analysis of factors associated with urinary retention morphine equivalent dose on the day of catheter removal was associated strongly with POUR. These results are statistically inconsistent. The study was retrospective, and POUR was defined as an inability to void within eight hours after catheter removal and not based on the actual measurement of the urinary bladder volume. The literature review reveals no evidence-based data supporting an eight-hour follow-up of postoperative urinary retention [5].

In our study, $33 \%$ of the patients developed POUR, which is the same rate as in previous studies [2, 4, 5]. Male gender and duration of the surgery were associated with POUR, which has been shown in other studies as well [4, 9]. The cumulative consumption of opioids during the hospital stay and on the day of catheter removal was directly associated with development of POUR in our study. Opioids have been shown to induce POUR, but the exact mechanism is not well understood [1, 7].

Spinal surgery has special complication risks. Correction of the spinal deformity has effects on the spinal cord and nerve roots. Manipulation of these neural elements may result in nerve or spinal cord deficits. In our study, all patients had spinal cord monitoring. Only one patient had a mild neurological deficiency postoperatively. Which was corrected by Th5 screw change, one week after initial surgery. The number of fusion levels nor extending the fusion into the lumbar spine did not have any impact on POUR. In the current study intraoperative pedicle screw replacement did not predict higher incidence of POUR. This observation indicates that direct mechanical compression may not play a central role in the development of urinary retention.

POUR is a clinically important issue with multifactorial aetiology and poor existing guidelines for definition and management in the paediatric population. The incidence of POUR is high among AIS patients after surgery and effort should be taken to reduce retention. Opioid consumption is one risk factor and needs to be minimized by multimodal pain management. Improvements to our current protocol could be use of regional analgesia such as erector spinae block [17] and an extended use of nonsteroidal anti-inflammatory drugs, since risk of non-union is low in the paediatric population.

\section{Strengths and limitations}

The study was a retrospective analysis of a prospectively collected paediatric spine register based data on a large cohort of homogenous AIS patients. These patients are generally healthy and do not have any difficulties in urinary bladder function. All patients were operated by the same two experienced orthopaedic spine surgeons. All patients had postoperative care in the paediatric intensive care unit at least for one night. The multimodal pain management included either oxycodone PCA or intravenous and oral oxycodone during the first $48 \mathrm{~h}$ postoperatively. The removal of urinary catheter included standardized measurement of the residual volume after initial voiding and data collection on voiding difficulties.

\section{Conclusions}

POUR affects one third of AIS patients after PSF, and higher postoperative opioid consumption increases the risk of urinary retention in these patients. Additional risk factors include longer duration of surgery, male gender, and increasing weight. POUR is associated with a number of risks and complications and may lead to unnecessary distension of the detrusor muscles. Postoperative urinary retention should be prevented in AIS patient undergoing PSF, especially by minimizing the need of opioids by multimodal analgesia. Postoperative urinary retention should be screened in this patient population with ultrasound and treated promptly with catheterization when diagnosed.

Authors' contributions TY conceived and designed the study, acquired, analysed, and interpreted the data, wrote the manuscript. LH conceived and designed the study, acquired, analysed, and interpreted the data, statistical analysis, critically revised the manuscript. MT conceived and designed the study, critically revised the manuscript. HO acquired the data, critically revised the manuscript. HK acquired the data, critically revised the manuscript. TK carried out the statistical analysis, critically revised the manuscript. IH conceived and designed the study, acquired the data, critically revised the manuscript.

Funding Open access funding provided by University of Turku (UTU) including Turku University Central Hospital. Scientific funding obtained from Turku University Hospital, The Finnish Society of Anaesthesiologists, Turku University Hospital Research Foundation, Medtronic, Stryker Spine and Paediatric Research Foundation. The funding body did not play a role in the investigation or writing of the 
manuscript. The funds were only used for salary for research nurse and funding research leaves.

Availability of data and material The data generated and analysed during the current study are available from the corresponding author on reasonable request.

\section{Declarations}

Conflict of interest Author Ilkka Helenius has been working as a consultant for Medtronic and K2M.

Ethical approval The study was approved by the Ethics Committee of Turku University Hospital District.

Consent to participate Informed consent has been obtained from the participants, and data have been collected in paediatric spine register.

Open Access This article is licensed under a Creative Commons Attribution 4.0 International License, which permits use, sharing, adaptation, distribution and reproduction in any medium or format, as long as you give appropriate credit to the original author(s) and the source, provide a link to the Creative Commons licence, and indicate if changes were made. The images or other third party material in this article are included in the article's Creative Commons licence, unless indicated otherwise in a credit line to the material. If material is not included in the article's Creative Commons licence and your intended use is not permitted by statutory regulation or exceeds the permitted use, you will need to obtain permission directly from the copyright holder. To view a copy of this licence, visit http://creativecommons.org/licenses/by/4.0/.

\section{References}

1. Baldini G, Bagry H, Aprikian A, Carli F, Phil M (2009) Postoperative urinary retention anesthetic and perioperative considerations. Anesthesiology 110:1139-1157. https://doi.org/10.1097/ ALN.0b013e31819f7aea

2. Boulis NM, Mian FS, Rodriguez D, Cho E, Hoff JT (2001) Urinary retention following routine neurosurgical spine procedures. Surg Neurol 55:23-27. https://doi.org/10.1016/S0090-3019(01) 00331-7

3. Altschul D, Kobets AJ, Nakhla J et al (2017) Postoperative urinary retention in patients undergoing elective spinal surgery. J Neurosurg Spine 26:229-234. https://doi.org/10.3171/2016.8.SPINE 151371

4. Keskinen H, Helenius L, Pajulo O, Helenius IJ (2018) Postoperative urinary retention or difficulties to empty the bladder in young patients undergoing posterior spinal fusion for adolescent idiopathic scoliosis. J Pediatr Surg 53:1542-1546. https://doi.org/ 10.1016/j.jpedsurg.2017.09.023

5. Sherburne E, Sawin K (2008) Investigating time to void after lower-extremity orthopedic surgery in a pediatric population. JSPN 13:36-47. https://doi.org/10.1111/j.1744-6155.2008. 00132.x
6. Rosseland LA, Stubhaug A, Breivik H (2002) Detecting postoperative urinary retention with an ultrasound scanner. Acta Anaesthesiol Scand 46:279-282. https://doi.org/10.1034/j.1399-6576. 2002.t01-1-460309.x

7. de Boer HD, Detriche O, Forget P (2017) Opioid-related side effects: postoperative ileus, urinary retention, nausea and vomiting, and shivering. A review of the literature. Best Pract Res Clin Anaesthesiol 31:499-504. https://doi.org/10.1016/j.bpa.2017.07. 002

8. Austin PF, Bauer SB, Bower W et al (2016) The standardization of terminology of lower urinary tract function in children and adolescents: Update report from the standardization committee of the International Children's Continence Society. Neurourol Urodyn 35:471-481. https://doi.org/10.1002/nau.22751

9. Knight BA, Bayne AP, Zusman N, Barney N, Yang S (2020) Postoperative management factors affect urinary retention following posterior spinal fusion for adolescent idiopathic scoliosis. Spine Deform 8:703-709. https://doi.org/10.1007/s43390-020-00090-9

10. Jackson J, Davies P, Leggett N et al (2019) Systematic review of interventions for the prevention and treatment of postoperative urinary retention. BJS open 3:11-23. https://doi.org/10.1002/bjs5. 50114

11. Golubovsky JL, Ilyas H, Chen J, Tanenbaum JE, Mroz TE, Steinmetz MP (2018) Risk factors and associated complications for postoperative urinary retention after lumbar surgery for lumbar spinal stenosis. Spine J 18:1533-1539. https://doi.org/10.1016/j. spinee.2018.01.022

12. Hresko MT (2013) Idiopathic scoliosis in adolescents. N Engl J Med 368:834-841. https://doi.org/10.1056/NEJMcp1209063

13. Hooton TM, Bradley SF, Cardenas DD et al (2010) Diagnosis, prevention, and treatment of catheter-aassociated urinary tract infection in adults: 2009 international clinical practice guidelines from the infectious diseases society of America. Clin Infect Dis 50:625-663. https://doi.org/10.1086/650482

14. Nevéus T, von Gontard A, Hoebeke P et al (2006) The standardization of terminology of lower urinary tract function in children and adolescents: report from the standardisation committee of the International Children's Continence Society. J Urol 176:314-324. https://doi.org/10.1016/S0022-5347(06)00305-3

15. Poyhia R, Seppala T, Olkkola KT, Kalso E (1992) The pharmacokinetics and metabolism of oxycodone after intramuscular and oral administration to healthy subjects. J Clin Pharm 33:617-621. https://doi.org/10.1111/j.1365-2125.1992.tb04090.x

16. Kalso E (2005) Oxycodone. J Pain Symptom Manage 29:47-56. https://doi.org/10.1016/j.jpainsymman.2005.01.010

17. Ma J, Bi Y, Zhang Y, et al (2021) Erector spinae plane block for postoperative analgesia in spine surgery: a systematic review and meta-analysis. Eur Spine J. https://doi.org/10.1007/s00586-02106853-w (published online: 13 May 2021)

Publisher's Note Springer Nature remains neutral with regard to jurisdictional claims in published maps and institutional affiliations. 